\author{
Marija Pejić* \\ Università di Belgrado
}

\title{
LA LETTURA DEL I CANTO DEL PURGATORIO
}

\begin{abstract}
Analizzando il brano introduttivo del I canto, l'autrice si sofferma sulla modalità dell'uso e sulla struttura della figura retorica apostrofe. Tenendo presente che il poeta innova e reinterpreta i modelli classici e che nella Commedia applica i procedimenti particolari anche a questa figura, già trasformata da un semplice dispositivo retorico in "figura nobile" nei testi delle auctoritas, nel presente contributo esaminerà in particolare uno degli esempi di tali procedimenti, dove la sottocategoria dell'apostrofe, la captatio benevolentiae, viene impiegata per strutturare la compagine del testo, ovvero il I canto del "Purgatorio", dandogli la valenza poetica e filosofico-retorica.

Parole chiave: figure stilistiche, modello retorico, captatio benevolentiae, Purgatorio, reinterpretazione.
\end{abstract}

Il primo canto del Purgatorio, già introdotto come luogo "diverso" dall'ultima parola della cantica precedente, benché rappresenti una specie di ritorno dal punto di vista formale alla struttura del proemio della prima cantica, si apre con un inconfutabile distacco dai versi infernali. Oltre ai cambiamenti sul piano strutturale introdotti tramite la descrizione di un ambiente nuovo, più quieto e promettente, il poeta intraprende il viaggio per le "miglior acque" mettendo subito in questione alcune concezioni classiche, soprattutto quelle relative alla retorica, nello stesso modo in cui l'aveva fatto all'apertura dell'Inferno. Consapevole, come noto, della propria modernità rispetto ai predecessori illustri, Dante non di rado esalta sé stesso e la propria maestria retorica, tanto che, grazie ad alcuni personaggi della Commedia, come Alessio Interminelli, ma soprattutto Ulisse (Padoan 1987), il suo elaborato sistema retorico diventa ancor più complesso. La questione si riflette innanzitutto nella problematizzazione del principio rethorica musicaque poita stabilito nel De vulgari eloquentia, che ora diventa il punto di partenza nel tentativo del poeta di inglobare, nel linguaggio poetico-nar-

*pejic_marija@yahoo.com 
rativo, i mezzi efficaci della retorica giuridica. All'entrata nel Purgatorio ${ }^{1}$, per ragioni già dette, il poeta esplicitamente torna alle questioni retoriche aperte, soprattutto nell'episodio dell'incontro con Catone Uticense. Nella forma di un lungo discorso che Virgilio rivolge a Catone $(P g, \mathrm{I}, 52-84)$, con l'apostrofe come la figura portante, questo episodio rappresenta anche l'ultimo esempio della captatio benevolentiae nell'opera e così diventa una cornice perfetta entro la quale Dante mette insieme alcuni concetti già espressi prima e dove addopera tutto l'aparato critico-retorico in funzione dell'analisi del testo.

Subito dopo l'apertura della nuova cantica, con una specifica invocazione delle muse ${ }^{2}$ e una breve descrizione del nuovo, luminoso ambiente in cui si è trovato (il simbolo della speranza da sé), Dante porta in scena il personaggio di Catone ${ }^{3}$. Alla stregua di altri personaggi della Commedia, lui non rivela esplicitamente la propria identità, né il suo nome viene indicato nel testo, ma sono le parole di Virgilio che delineano chiaramente, nella sapiente costruzione del discorso, il personaggio di Catone di Utica, passato, come lo sa Dante grazie alle fonti, alla storia come il simbolo dell'avversario della tirannia che, dopo la caduta della repubblica, si suicida perché non vuole rinunciare alle proprie virtù morali e ai propri diritti civilii ${ }^{4}$ Ma, è la

${ }^{1}$ Riguardo l'inizio del Purgatorio in: Barberi Squarotti (1970), Getto (1984), Bigi (1970), Chiari (1966), Illiano (1977), Raimondi (1963) e Bosco (1967).

2 Sull'importanza delle invocazioni nella Commedia: Hollander (2013), Contini (1970), Battaglia Ricci (1983), Hollander (1969), Auerbach (1984) et al.

${ }^{3}$ Prima di tutto, occorre tener presente che Dante comincia ogni cantica della Commedia con una forma dell'apostrofe, ovvero invocatio musae. Formalmente celato dietro un velo della retorica tradizionale, l'autore adatta e avvicina al pubblico alcuni principi e concetti della dottrina cristiana adeguando la classica invocatio al sermo humilis medievale (Garavelli 2003), cambiandolo gradualmente e avvicinandolo sempre di più alle nuove richieste culturali ed ideologiche, ma anche a quelle poetiche che si rispecchiano in una specifica applicazione del principio della convenientia. Ogni "invocazione nuova" (a parte quella di Paradiso) sarà seguita da una rimodellata captatio benevolentiae. Così Dante, infatti, apre la sua opera non soltanto con una, ma con addirittura due forme apostrofiche. In tal modo prepara il suo pubblico alle descrizioni crudeli e terrificanti del mondo ultraterreno, mentre contemporaneamente entra in un dialogo specifico con la tradizione letteraria, evidenziando l'indiscutibile originalità della propria opera, così come l'importanza della retorica per la sua scrittura (Barberi Squarotti 1970).

${ }^{4}$ Non solo nell'antichità, ma anche durante tutto il medioevo, Catone diventa il simbolo della virtù civile e della persistenza. Di più, il suo atto di suicidio differenzia abbastanza da quello che compiono i peccatori del XIII canto d'Inferno, visto che non rappresenta il "prodotto" finale di una vita trascorsa nel peccato e di debolezza dell'anima, ma, al contrario, esprime l'elevatezza e la purezza d'animo, la nobiltà di altissimo livello dei tempi antichi (Hollander 2010). Anche alcuni padri della Chiesa, invece, come 
descrizione stessa del nuovo personaggio, così come la reazione di Virgilio alla sua apparizione, un segnale che non si tratti di un'anima qualsiasi. Dante rappresenta Catone come un vecchio canuto con la barba e i capelli lunghi, usando l'appellativo veglio (fr. ant. vieil) che appartiene al registro mediano del Purgatorio, a differenza del termine veculum (lat. pop.) che usa frequentemente nell'Inferno (III, 83) o del latinismo senem adatto al registro sublime del Paradiso (XXXI, 59) (Bigi 1970). Lui, con l'accusa rivolta ai due poeti di cercare ad entrare di nascosto nel Purgatorio ${ }^{5}$, apre il primo dialogo della cantica e si qualifica, da solo, come il guardiano del purgatorio $^{6}, \mathrm{ma}$, a differenza di guardiani precedenti, figure diaboliche, un guardiano peccatore degno della grazia divina, anzi, il guardiano della grazia divina? ${ }^{7}$. Il che non solo provoca il cambiamento dell'atteggiamento di Virgilio nei confronti dei guardiani - e di conseguenza anche quello di Dante viaggiatore - ma suggerisce anche il "salto di qualità" del dialogo, ovvero la necessità di argomentare in altro modo l'ineluttabilità del viaggio dantesco:

Lo duca mio allor mi diè di piglio, e con parole e con mani e con cenni reverenti mi fé le gambe e 'l ciglio, $P g$, I, 49-51.

Dimostrando così a Catone di essersi sbagliato sul loro conto, Virgilio prende la parola e risponde all'accusa:

Sant'Agostino (Sant'Agostino, De civitate dei contra paganos, I, 17, 20, 25) interpretavano il suicidio di Catone in chiave della virtù morale considerandolo, nello stesso tempo, un peccato: "De morte voluntaria ob metum poenae sive dedecoris; Nullam esse auctoritatem, quae Christianis in qualibet causa ius voluntariae necis tribuat; Quod peccatum non per peccatum debeat declinari”.

${ }^{5}$ Il richiamo va al primo discorso esortativo della Commedia che è quello tenuto da Virgilio e rivolto al poeta fiorentino, molto diverso da questo catoniano sia dal punto di vista retorico, in quanto alla sua funzione. Di più sull'argomento vedi: Marcozzi (2017), Battistini (2013), Gorni (2002), Getto (1960) e Giannantonio (1980).

6 "“Chi siete voi che contro al cieco fiume / fuggita avete la pregione etterna?', / diss'el, movendo quelle oneste piume. // 'Chi v'ha guidati, o che vi fu lucerna, / uscendo fuor de la profonda notte / che sempre nera fa la valle inferna? // Son le leggi d'abisso così rotte? / o è mutato in ciel novo consiglio, / che, dannati, venite a le mie grotte?'”, $P g$, I, 38-48.

7 “'...] vidi presso di me un veglio solo, / degno di tanta reverenza in vista, / che più non dee a padre alcun figliuolo. // Lunga la barba e di pel bianco mista / portava, a' suoi capelli simigliante, / de' quai cadeva al petto doppia lista. // Li raggi de le quattro luci sante / fregiavan sì la sua faccia di lume, / ch'i' '1 vedea come '1 sol fosse davante", $P g$, I, 31-9. 
Poscia rispuose lui: 'Da me non venni: donna scese del ciel, per li cui prieghi de la mia compagnia costui sovvenni.

Ma da ch'è tuo voler che più si spieghi di nostra condizion com'ell'è vera, esser non puote il mio che a te si nieghi.

Questi non vide mai l'ultima sera ma per la sua follia le fu sì presso, che molto poco tempo a volger era.

Si com'io dissi, fui mandato ad esso per lui campare; e non lì era altra via che questa per la quale i'mi son messo.

Mostrata ho lui tutta la gente ria e ora intendo mostrar quelli spirti che purgan sé sotto la tua balia.

Com 'io l'ho tratto, saria lungo a dirti; de l'alto scende virtù che m'aiuta conducerlo a vederti e a udirti.

$P g$, I, 52-84.
Or ti piaccia gradir la sua venuta: libertà va cercando, ch'è sì cara, come sa chi per lei vita rifiuta.

Tu'l sai, ché non ti fu per lei amara in Utica la morte, ove lasciasti la vesta ch'al gran dì sarà sì chiara.

Non son li editti etterni per noi guasti ché questi vive e Minòs me non lega ma son del cerchio ove son li occhi casti

di Marzia tua, che ' $n$ vista ancor ti priega o santo petto, che per tua la tegni:

per lo suo amore adunque a noi ti piega.

Lasciane andar per li tuoi sette regni; grazie riporterò di te a lei, se d'esser mentovato là giù degni',

Virgilio è consapevole che si trova in una posizione ingrata, ossia si rende conto che la sua risposta deve adempiere necessariamente la funzione duplice. Da un lato, come in precedenza, deve mettersi in difesa di Dante viaggiatore e assicurargli il passaggio. Dall'altro, però, trovandosi davanti alla porta del nuovo regno ultraterreno al quale non appartiene e che non capisce pienamente, cerca anche di difendere sé stesso, il proprio ruolo di guida e di autorità che già qui comincia a vacillare. In altre parole, per poter soddisfare diversi obiettivi simultaneamente, Virgilio sarà costretto ad organizzare il suo discorso servendosi del mezzo "sicuro", ovvero della retorica classica nella quale è il maestro per eccellenza, combinando e reinterpretando diverse formule stilistiche. Sul piano retorico, dunque, la combinazione dei diversi modelli comporta la combinazione di stili, ovvero di corrispondenti modelli espressivi ${ }^{8}$. Comincia a parlare con molta cau-

${ }^{8}$ Come possiamo notare nel testo, Dante usa questo procedimento spesso. L'uso di molte figure basate sul collegamento di due concezioni tramite asindeto o polisindeto (comparatio, antitesis, similitudo ecc.) diventa uno degli strumenti principali con cui costruisce il discorso realistico, insistendo su alcune relazioni spazio-temporali, così come sulle immagini plastiche di alcuni personaggi dell'opera. Visto che alla base di ogni 
tela usando il registro medio-alto, organizzando il suo discorso secondo le regole della difensoria e dell'orazione epidittica, fondendo i loro elementi per rendere il proprio monologo chiaro e preciso e per allontanare ogni sospetto, o almeno moderare la sentenza. È, inoltre, carico d'emozioni, dato che Catone non svolge soltanto il ruolo del procuratore, ma anche quello di giudice e d'intera giuria.

Virgilio già dalla prima terzina fa notare che è investito di una missione santa ("da me non venni") e aumenta il proprio valore con un sillogismo ("donna scese del ciel, per li cui prieghi / de la mia compagnia costui sovvenni') abbassando l'enfasi del testo subito dopo, nella seconda terzina, preparando la scena per presentare la difesa ${ }^{9}$. Nelle tre terzine successive offre alcune risposte elementari sul perché Dante si è trovato in inferno, perché lo accompagna e gli mostra il mondo ultraterreno, alludendo costantemente all'ineluttabilità e alla predestinazione del loro viaggio, invocando così in aiuto l'autorità celeste.

Poi cambia improvvisamente il tipo dell'orazione e dalla classica auguratio passa alla captatio benevolentiae sotto forma dell'insinuatio in senso stretto ("com'io l'ho tratto, saria lungo a dirti; / de l'alto scende virtù che m'aiuta / condurcerlo a vederti e a udirti"), ommettendo appositamente i fatti potenzialmente sfavorevoli, per alludere al dubbio se il compito assegnatogli da Beatrice possa essere giudicato svolto adeguatamente. Perciò intenzionalmente lascia la sua orazione incompleta e aperta reindirizzando

allegoresi sta lo schema della figura transumptio e translatio (metalesi) dove il significato viene trasmesso da un termine ad un altro, diventa evidente che la metafora e l'allegoria, insieme ad altre figure simili (simbolo, sineddoche ed al.), rappresenta uno dei mezzi retorici più utilizzati nel testo della Commedia. Ciononostante, poiché entrambe appartengono ai tropi, l'espressione metaforica riguarda necessariamente soltanto una delle dimensioni del testo. Proprio per questo apostrofe, la figura nobilis che già nell'antichità acquisiva le caratteristiche di un paradigma (Of Vinsauf 1967), verrà imposta come modello ideale per la costruzione dell'espressione figurativa. Di più su questo tema in: Marcozzi (2017), Garavelli (2003), Of Vinsauf (1967), Raimondi (1980), Bigi (1981), Nencioni (1983), Tateo (1995) et al.

${ }^{9}$ È già noto che Dante gioca con l'intensità dell'azione drammatica e con l'enfasi del testo indirizzando l'attenzione dei lettori verso quelle parti dell'episodio che considera rilevanti. Ciò di solito ottiene interrompendo bruscamente la narrazione, portando sulla scena un nuovo personaggio che si rivolge agli altri usando sempre una delle figure retoriche estremamente enfatiche, soprattutto l'apostrofe. Di più in: Auerbach (1984), Beccaria (2013), Getto (1955) et al. Però, è significativo che questo compito non viene mai affidato al personaggio di Virgilio. Non solo il poeta latino interromperà la narazione molto di rado, ma in poche occasioni che lo fa non adopererà quasi mai l'apostrofe, scegliendo una delle figure meno "drammatiche" come comparatio, antitesis, similitudo ecc. Di più in: Auerbach (1984), Beccaria (2013), Marcozzi (2017) et al. 
l'attenzione a Catone e incominciando a lodarlo smisuratamente. Così cambia gradualmente la forma del discorso e mette in relazione Dante con Catone, con l'accento sul motivo della libertà, un concetto, tra l'altro, caro a Catone ("or ti piaccia gradir la sua venuta: / libertà va cercando, ch'è sì cara, / come sa chi per lei vita rifiuta"). In tal modo le parole di Virgilio assumono un tono sempre più personale che culminerà nelle terzine seguenti. Precisando il posto e le circostanze che hanno portato Catone al peccato, Virgilio lo raffigura in soli tre versi, giustificando allo stesso tempo i suoi peccati e mettendo in evidenza che dopo il giudizio finale si eleverà in cielo tra i beati. Poi in modo chiaro e conciso, in qualità del difensore, pronuncia l'ultima parola, ovvero sottolinea il fatto inconfutabile che Dante viaggiatore è ancora vivo e che lui stesso deve scontare la sua condanna nel limbo e che, quindi, nessuna legge divina è stata effettivamente violata ("non son li editti eterni per noi guasti / ché questi vive e Minòs me non lega"). Con l'ultimo verso di questa terzina Virgilio cercherà di convalidare la sua difensoria e così le due successive rappresenteranno il culmine della sua orazione a forma di una captatio in senso stretto estremamente enfatizzata. Per ottenere la simpatia di Catone, il poeta latino giocherà la sua carta vincente, ossia cercherà di fare riferimento a Marzia, la moglie di Catone, con la promessa di ringraziarlo anche di fronte a lei, sempre se le leggi del Limbo lo consentono ${ }^{10}$.

La scelta della captatio benevolentiae come mezzo persuasivo principale del discorso non sorprende molto, dato che fa parte del campo retorico dell' apostrofe (Arduini e Damiani: 2010) ${ }^{11}$ la quale, con la sua forma aperta e la caratteristica di mettere in contatto diretto la dimensione orizzontale con quella verticale del testo, ovvero di mostrare una complessa relazione tra res e verba, a Dante verrà imposta come modello ideale per la costruzione dell'espressione figurativa della Commedia (Garavelli 2003). Tutto

${ }^{10}$ Di più sul tema del Limbo: Rossi (2013), Padoan (1970) et al.

${ }^{11}$ Con l'appellativo "nobilis figura", ma grazie anche alla sua capacità di espandere ed arricchire l'azione drammatica (amplificatio), ossia di sintetizzarla e semplificarla (abbreviato), facendo un effetto particolare sul pubblico muovendo efficientemente le sue emozioni (movere) e suscitando il pathos, l'apostrofe si trasmetterà dall'apparato retorico classico e si inserirà nel nuovo sistema medievale del sermo humilis diventando, nello stesso tempo, un mezzo retorico estremamente adatto alla poesia sublime. Tuttavia, l'apostrofe medievale non deve essere percepita soltanto come una figura, ma più come una dimensione o un paradigma retorico che unisce in sé un intero spettro delle figure e dei procedimenti stilistici. Con le funzioni discorsive sono ben precisate e l'obiettivo principale di manipolare e regolare l'enfasi del testo, essa supera i limiti dell'amplificatio al quale è formalmente subordinata. Di più su questo tema in: Garavelli (2003), Arduini e Damiani (2010), Tateo (1995) et al. 
nuovo è, però, il modo in cui Dante adopera la classica captatio $^{12}$ giuridica usandola non solo per orientare sia l'attenzione che le emozioni dell'interlocutore, ma anche in qualità di una cornice perfetta per esprimere nuove concezioni cristiane. Questa captatio, straordinariamente sonora, rafforzata con un'apostrofe enfatica e con i verbi priega/piega grazie ai quali riesce quasi a riprodurre l'effetto della preghiera cristiana, senza alcun dubbio assomiglia molto a quella di Beatrice del canto II d'Inferno. Si potrebbe senz'altro affermare che la difensoria virgiliana viene idealmente ispirata dall'orazione di Beatrice. Per quanto riguarda la struttura, le due orazioni sono praticamente identiche, formalmente costruite sul modello dell'apostrofe in lode. Durante il discorso avviene un'alternanza frequente dei versi concisi, usati ogni volta che diventa necessario spiegare qualcosa o ricordarsi di qualche inevitabilità, e quelli poetici, teneri con cui si riconquista l'attenzione dell'interlocutore. Di più, concepito su due modelli di captatio benevolentiae, il discorso viene anche rinforzato con l'apostrofe come mezzo retorico dominante con cui si ottiene il pathos finale. Tra Virgilio e Beatrice viene stabilita la relazione della maestra e lo studente (Grimaldi 2017), una relazione che si apre con una captatio autoritativa, ma soave della donna beata all'inizio dell'Inferno e si conclude con la figura premurosa dell'antico poeta alle soglie dell'impero a cui non appartiene, dove con ogni passo perde la forza di prima.

Rileggendo alla luce del discorso trasposto di Beatrice le parole di Virgilio, che riflettono rettamente le sue, si sottolinea l'insuperabile divario tra questi due esempi. Beatrice, infatti, non vuole semplicemente persuadere Virgilio e la sua captatio, quindi, rispecchiando lo schema dell'antica prassi retorica, serve come una cornice conveniente a rappresentare certi concetti cristiani e così diventa il perfetto esempio della captatio cristianizzata. Dall'altra parte, la volta che dovrà affrontare Catone, Virgilio si mostrerà maldestro ad emulare il modello oratorio della donna beata. Adottando solo la struttura formale della sua captatio ed insistendo sulla persuasio, il poeta organizzerà la propria orazione trascurando quasi del tutto il contesto cristiano. Il modus parlandi del poeta che combacia il suo modus pensandi si dimostrerà di nuovo l'elemento distintivo del suo personaggio, così come il suo continuo tentativo di superare la cornice retorica antica per accedere, almeno a parole, alla grazia divina. Effettivamente, la sua impresa finisce ingloriosamente - Catone non solo rimane insensibile alla sua orazione e dubbioso riguardante la verità delle sue parole, ma perfino lo rimprovera

${ }^{12}$ Di più sulla captatio benevolentiae classica e sulla sua trasformazione dalla tecnica retorica al topos letterario, così come sulle sue subcategorie principium o insinuatio in senso stretto vedi in: Garavelli (2003) e Arduini e Damiani (2010). 
esplicitamente, rilevando il fatto che Virgilio non comprende pienamente il nuovo concetto cristiano:

'Marzia piacque tanto a li occhi miei mentre ch 'i'fu'di là', diss'elli allora, 'che quante grazie volse da me, fei.

Or che di là dal mal fume dimora, più muover non mi può, per quella legge che fatta fu quando me n'usci'fora.

Ma se donna del ciel ti muove e regge, come tu di', non c'è mestier lusinghe: bastisi ben che per lei mi richegge',

$P g$, I, 85-93.

Se in diversi luoghi dell' Inferno incontriamo diverse indicazioni della presunta onnipotenza di Vergilio, come i viaggiatori si avvicinano al colle della purificazione, sempre di più la sua capacità di comprensione diventa più fragile (Hollander 1983). Con le parole di Catone si dimostra esplicitamente che Virgilio, in quanto simbolo della ragione umana, non sarà più capace d'interpretare da solo il nuovo regno divino, dato che alla sua soglia non solo dimentica le regole celesti, ma le viola cercando d'ingannare Catone con le lusinghe. La mancanza della consapevolezza si legge anche dalla mancanza di alcuna sua reazione: dopo che Catone improvvisamente sparisce, nello stesso modo in cui è apparso sulla scena, e dopo che Dante si alza silenzioso guardando dritto il poeta aspettando una spiegazione, Virgilio deluderà le sue aspettative rivolgendosi al fiorentino con una voce tenera, quasi supplichevole, incoraggiando Dante a seguirlo ${ }^{13}$ :

«Figliuol, segui i miei passi:

volgianci in dietro, ché di qua dichina

questa pianura a'suoi termini bassi»,

$P g, \mathrm{I}, 112-4$.

${ }^{13}$ Dopo questo episodio, diventano sempre più frequenti gli episodi in cui si manifesta l'incapacità di Virgilio per capire l'essenziale della dottrina cristiana a partire dal canto successivo dove Cato riappare sulla scena per rimproverare fortemente tutte le anime che stanno ascoltando la canzone di Casella e dove si possono notare per la prima volta i segni di rimorso da Virgilio: "El mi parea da sé stesso rimorso: / o dignitosa coscienza e netta, / come t'è picciol fallo amaro morso!"' $P g$, III, 7-9, ma anche le prime tracce di sospetto di Virgilio nascoste sotto il velo delle dolci lodi: "I' mi ristrinsi a la fida compagna: / e come sare' io sanza lui corso? / chi m'avria tratto su per la montagna?" $P g$, III, 4-6, per culminare con l'apparizione d'un'altra guida, Stazio e la scomparsa di Virgilio in Eden. 
Quindi, il personaggio di Virgilio viene messo in primis in rapporto con Dante viaggiatore, incarnazione di un peccatore medievale che pur appartenendo al concetto della dottrina cristiana riesce ad accettare ed a concepire la tradizione antica. Di conseguenza, si istituisce il raffronto con il personaggio di Catone, il rappresentante ideale dell'antichità riformata $\mathrm{e}$ cristianizzata, capace d'intendere e di applicare le leggi divine. Il tertium comparationis è la donna beata, come abbiamo visto, in cui si personificano i principi teologico-dogmatici basilari del nuovo pensiero cristiano. In tal modo diventa più evidente la maniera in cui la figura di Virgilio oltrepassa le soglie dell'allegoria e ottiene la profondità umana, simile a quella solita a Dante viaggiatore. Riaffermandosi un personaggio a tutto tondo, il poeta latino riconferma un'ultima volta anchè la propria collocazione nel Limbo, tra i poeti di "bella scuola" 14 .

Con la formula captatio benevolentiae Dante, dunque, determinerà le regole in cui la tradizione classica può e deve incidere sulla dottrina cristiana, limitando la funzione di Virgilio alla materia e allo stile medio-bassi (Hollander 1983). Evidenzia, inoltre, un ulteriore distacco dal personaggio di Beatrice che, anche se immagine del pensiero cristiano rivelato, unisce in sé il sapere di tutte le tradizioni precedenti. Ciò si conferma anche nella sua orazione autorevole dell'Eden, concepita di nuovo intorno al modello apostrofico, con la quale i due regni ultraterreni vengono finalmente definiti, ma dai quali il viaggiatore deve purificarsi persino letteralmente per poter entrare in quello sublime accompagnato da una guida nuova e meritevole (Desideri 2016). La captatio d'esito negativo che Virgilio rivolge a Catone non serve soltanto come un'ulteriore caratterizzazione del personaggio del poeta latino, ma diventa anche una formula specifica con cui si chiude l'Inferno e, di conseguenza, con la quale si apre il Purgatorio. Con la captatio, inoltre, tramite un costante confronto delle concezioni antiche e cristiane, vengono di nuovo sottolineate le differenze tra due mondi ultraterreni non soltanto sul livello teologico e ideologico, morale ed etico, ma anche su quello stilistico e retorico. L'essenziale nell'episodio iniziale del Purgatorio non è, quindi, soltanto la condanna di Catone della vecchia tradizione incapace di concepire il nuovo modello cristiano. L'attenzione si sposta anche sull'atteggiamento della antichità nei confronti della nuova cultura cristiana. Rimproverando Virgilio, le parole del poeta vengono definite di conseguenza come lusinghe e così anche la bravura della retorica antica viene rappresentata come seduttrice ed ingannevole ed intera oratoria tradizionale viene caratterizzata come negativa e peccatrice. Se la problematica della concezione della retorica si apriva con una nuova connotazione di Ulisse,

${ }^{14}$ Di più riguardo questo tema in: Iannucci (1993), Hollander (1983), Chiesa (2017) e Comparetti (1981). 
ora sull'esempio della fallita captatio di Virgilio la retorica si afferma finalmente come un' arte negativa, ingannevole ed indegna del regno celeste. Allo stesso tempo, perfino la posizione dell'intera poesia sarà messa in dubbio. Perciò nel Purgatorio, dopo il rimprovero di Catone, il modello della poesia religiosa innica verrà gradualmente stabilito come l'unica forma adatta per la poesia sublime.

Di conseguenza, insieme alla retorica, verrà messa in dubbio l'intera concezione antica del mondo che ora deve essere purificato e trasformato, privato di tutte le caratteristiche contrastanti ai principi del nuovo codice cristiano. Se Dante viaggiatore dovrà purgarsi dal deposito infernale, Dante autore dovrà, però, adeguare il proprio stile ai nuovi versi, attenuando le "asprezze" e "chioccezze" caratteristiche per la materia infernale. Così non pare strano che poeta non aprirà il Paradiso con un'altra formula di captatio benevolentiae, che si trova, però, all'inizio di altre due cantiche. Uno stile più pacato ed elevato prenderà sempre di più il primato, affinché culmini pienamente con la scomparsa graduale non solo della captatio benevolentiae, ma di tutte le figure retoriche dell'antica prassi giuridica, per dare spazio, nel Paradiso, alla nuova retorica del sermo humilis cristiano - una retorica che non si depriva del ricco repertorio dei mezzi reotici classici, ma li reinterpreta e adegua alla nuova visione del mondo medievale.

\section{BIBLIOGRAFIA}

Arduini, S. e Damiani, M. (2010). Dizionario di retorica. Covilhã: Livros LabCom.

Auerbach, E. (1984). Studi su Dante. Milano: Feltrinelli.

Barberi Squarotti, G. (1970). Ai piedi del monte. Il prologo del «Purgatorio».

In «Purgatorio» I, «Letture Classensi» 3. Ravenna: Longo.

Battaglia Ricci, L. (1983). Dante e la tradizione letteraria medievale. Pisa:

Giardini Editori.

Battistini, A. (2013). Canto I. Dalla paura alla speranza. In E. Malato e A.

Mazzucchi (a cura di), Cento canti per cento anni (pp. 43-74). Roma:

Salerno.

Beccaria, G. (2013). Ritmo e melodia nella prosa italiana. Studi e ricerche sulla prosa d'arte. Firenze: Olschki.

Bigi, E. (1970). Il canto I del Purgatorio. Letture dantesche. Firenze: Le Monnier

Bigi, E. (1981). Caratteri e funzioni della retorica nella "Divina commedia".

Forme e significati nella "Divina commedia” (pp. 185-203) Bologna:

Cappelli.

Bosco, U. (1967). Dante: il 'Purgatorio'. Torino: ERI. 
Chiari, A. (1966). Il preludio del 'Purgatorio'. Torino: Società Editrice Internazionale.

Chiesa, P. (2017). La letteratura latina del medioevo. Roma: Carocci.

Comparetti, D. (1981). Virgilio nel medioevo. Firenze: La nuova Italia.

Contini, G. (1970). Un'interpretazione di Dante. Varianti e altra linguistica (pp. 369-405). Torino: Einaudi.

Desideri, M. (2016). Dante e le "donne d'amore". Roma: Società Editrice Dante Alighieri.

Garavelli, B. M. (2003). Manuale di retorica. Roma: Bompiani.

Getto, G. (a cura di). (1955). Letture dantesche. Inferno. Firenze: Sansoni.

Getto, G. (1960). Il canto 1. dell' 'Inferno'. Firenze: Le Monnier.

Getto, G. (1984). L'Arte dell'interpretare: studi critici offerti a Giovanni Getto. Cuneo: Arciere.

Giannantonio, P. (1980). Canto 1. dell'Inferno. Napoli: Loffredo.

Gorni, G. (2002). Dante nella selva: il primo Canto della 'Commedia'. Firenze: F. Cesati.

Grimaldi, E. (2017). Il gioco della zara Dante tra Virgilio e Beatrice: alcune riflessioni. Pisa: ETS.

Hollander, R. (1969). Allegory in Dante's Commedia. Princeton: Princeton University Press.

Hollander, R. (1983). Il Virgilio dantesco. Firenze: Olschki.

Hollander, R. (2010). Ancora sul Catone dantesco. Letture dantesche. Firenze: SDI.

Hollander, R. (2013). Dante's nine invocations revisited. Princeton: Princeton University Press.

Iannucci, A. (a cura di). (1993). Dante e la "bella scola della poesia". Ravenna: Longo.

Illiano, A. (1977). Sulle sponde del prepurgatorio: poesia e arte narrativa nel preludio all'ascesa. Fiesole: Cadmo.

Marcozzi, L. (a cura di). (2017). Dante e la retorica. Ravenna: Longo Angelo.

Nencioni, G. (1983). Dante e la retorica. Tra grammatica e retorica. Torino: Einaudi.

Of Vinsauf, G. (1967). Poetria nova. Toronto: Pontifical Institute of Mediaeval Studies.

Padoan, G. (1970). Il Limbo dantesco. Letture Classensi 3 (pp. 187-217). Ravenna: Longo

Padoan, G. (1987). Il pio Enea, l'empio Ulisse: tradizione classica e intendimento medievale in Dante. Ravenna: Longo.

Raimondi, E. (1963). Il canto 1. del 'Purgatorio'. Firenze: F. Le Monnier.

Raimondi, E. (1980). Poesia come retorica. Firenze: Olschki. 
Rossi, L. C. (2013). Canto IV. Autoincoronazione poetica nel limbo. In E. Malato e A. Mazzucchi (a cura di), Cento canti per cento anni (pp. 131-161). Roma: Salerno

Tateo, F. (1995). Per dire d'amore: reimpiego della retorica antica da Dante agli arcadi. Napoli: Edizioni scientifiche italiane.

\author{
CAPTATIO BENEVOLENTIAE AS THE BASIC STRUCTURE \\ OF THE “NEW” DANTE'S RHETORICS - THE FIRST CANTO \\ OF THE PURGATORIO
}

\begin{abstract}
Summary
Analyzing the introductory passage of the first canto, the author of this paper focuses on the method of use and the structure of the rhetorical figure apostrophe. Having in mind that Dante is a writer who innovates and reinterprets the classic rhetorical models and that in the Comedy he applies his particular procedures even to the rhetorical figure of the apostrophe, already transformed from a simple rhetorical device into a "noble figure" in the texts of the auctoritas, in this paper the author analyses one of the examples of these procedures where the subcategory of the apostrophe, the captatio benevolentiae, is used for structuring the text, in our case, the first canto of the Purgatory, giving it the poetic, philosophical and rhetoric value.

Key words: stylistic devices, stylistic model, captatio benevolentiae, Purgatorio, reinterpretation.
\end{abstract}

